\title{
Alpha-catenin structure in solution and in complex with F-actin as revealed by small angle X-ray and neutron scattering
}

Iain D Nicholl, Tsutomu Matsui, Thomas M. Weiss, Christopher B. Stanley, William T. Heller, David J E Callaway, and Zimei Bu

City College of New York, CUNY

As a core component of the adherens junction, the F-actin binding protein $\alpha$-catenin stabilizes the cadherin/catenin complexes to the actin cytoskeleton for the mechanical coupling of cell-cell adhesion. Additionally, $\alpha$-catenin regulates actin dynamics, cell polarity and cell migration, functions that are independent of the adherens junction. We have determined the solution structures of $\alpha$-catenin monomers, dimers, and in complex to F-actin using in-line size-exclusion chromatography small-angle X-ray scattering (SEC-SAXS) and contrast-matching small angle neutron scattering (SANS). The solution structure of alpha-catenin is different from the crystal structure, in that the vinculin-binding $\mathrm{M}$ fragment and the actin-binding domain can adopt different configurations. When in complex to F-actin, the $\alpha$-catenin dimer has an open and extended conformation, and the $\alpha$-catenin assembled F-actin bundle develops into an ordered hexagonal arrangement. The results show that $\alpha$-catenin possesses a dynamic structure that primes itself as a mechanosensor protein. 